OPEN ACCESS

Edited by:

Akio Adachi,

Tokushima University, Japan

Reviewed by:

Nirupma Trehanpati,

Institute of Liver and Biliary Sciences,

India

Yu Shang,

Hubei Academy of Agricultural

Sciences, China

*Correspondence:

Ruiping She

sheruiping@126.com

tThese authors have contributed equally to this work.

Specialty section: This article was submitted to Virology,

a section of the journal

Frontiers in Microbiology

Received: 26 October 2017

Accepted: 27 February 2018

Published: 16 March 2018

Citation:

Yang Y, Shi R, Soomro MH, Hu F, Du F and She R (2018) Hepatitis E Virus Induces Hepatocyte Apoptosis via Mitochondrial Pathway

in Mongolian Gerbils.

Front. Microbiol. 9:460.

doi: 10.3389/fmicb.2018.00460

\section{Hepatitis E Virus Induces Hepatocyte Apoptosis via Mitochondrial Pathway in Mongolian Gerbils}

\author{
Yifei Yang ${ }^{1,2+}$, Ruihan Shi2t, Majid H. Soomro ${ }^{2}$, Fengjiao $\mathrm{Hu}^{2}$, Fang $\mathrm{Du}^{2}$ and Ruiping She ${ }^{2 \star}$ \\ ${ }^{1}$ Institute of Chinese Materia Medica, China Academy of Chinese Medical Sciences, Beijing, China, ${ }^{2}$ Laboratory of Animal \\ Pathology and Public Health, Key Laboratory of Zoonosis of Ministry of Agriculture, College of Veterinary Medicine, China \\ Agricultural University, Beijing, China
}

Previous studies demonstrated that Mongolian gerbils can be infected by hepatitis $\mathrm{E}$ virus (HEV), which induces the hepatic injury. Here, the mitochondria in hepatocytes from HEV-infected gerbils were considerably swollen, thin cristae. After HEV infection, the activity of superoxide dismutase significantly decreased $(p<0.01)$, while malondialdehyde concentrations significantly increased, compared with those in the control group $(p<0.01)$. Adenosine triphosphatase levels decreased significantly in the hepatocyte of the inoculated groups, compared with those in control group $(p<0.05)$ at days 21, 28, 42 post-inoculation (dpi) as well. Furthermore, the levels of ATP synthetase ATP5A1 significantly decreased during HEV infection, compared with those in the control group $(p<0.05)$. According to the TdT mediated dUTP nick end labeling (TUNEL) detection, TUNEL positive hepatocytes increased in the inoculated group, compared with that in the control group ( $p<0.05)$. Up-regulation of the mitochondrion-mediated apoptosis regulating proteins, Bax and $\mathrm{Bcl}-2$, in the HEV-infected gerbils $(p<0.05)$ was observed. However, cytochrome c levels in mitochondria decreased, while this molecule was detected in the cytoplasm of the infected animals, in contrast to that in the control group. Apaf-1, and active caspase- 9 and -3 levels were shown to be significantly higher in the inoculated group compared with those in the control group $(p<0.05)$. Taken together, our results demonstrated that HEV infection induces hepatocyte injuries and activity of the mitochondrial apoptotic pathway, which trigger the hepatocyte apoptosis in Mongolian gerbils.

Keywords: mitochondrion, hepatitis E virus, hepatocyte, apoptosis, injury

\section{INTRODUCTION}

Hepatitis $\mathrm{E}$ virus induces the development of hepatitis in many organisms, including humans. $\mathrm{HEV}$ is a non-enveloped, positive-sense, single-stranded 7.2-kb RNA virus belonging to the family Hepeviridae (Smith et al., 2014). It contains three ORFs (Panda et al., 2007), and HEVs isolated from humans, pigs, deer, wild boars, rabbits, camels, and mongooses belong to the

Abbreviations: ALT, alanine transaminase; AST, aspartate transaminase; ATP, adenosine triphosphate; Bax, Bcl2-associated $\mathrm{X}$ protein; dpi, day post-inoculation; HEV, hepatitis E virus; MDA, malondialdehyde; ORFs, open reading frames; SOD, superoxide dismutase; SPF, specific-pathogen free; TBIL, total bilirubin; TEM, transmission electron microscopy; TUNEL, TdTmediated dUTP nickend labeling. 
Orthohepevirus A group, while HEV isolated from chickens belongs to Orthohepevirus B, from rats to Orthohepevirus C, and from bats to Orthohepevirus D group (Smith et al., 2014). HEV infections represent an important public health problem and may lead to mortality and morbidity, especially in the developing countries.

Mitochondria are now recognized as the site of respirationdependent ATP synthesis. Furthermore, mitochondria fulfill many other important functions in eukaryotic cells and play a role in calcium storage, amino-acid metabolism, iron-sulfur cluster synthesis, lipid metabolism, and programmed cell death (Lill and Mühlenhoff, 2008; Martinou and Youle, 2011; Kulawiak et al., 2013).

Mitochondrial apoptosis pathway can be induced by mitochondrial injury and it is affected by the Bcl-2 family members (Degli Esposti et al., 2012; Wang, 2014). Ultimately, the effector caspases are activated, cleaving and degrading cell structures, which results in the release of the apoptotic products into the circulation (Wang, 2014). Viruses, drugs, and their reactive metabolites affect mitochondrial respiratory chain, causing a series of harmful responses, such as ATP depletion, mitochondrial DNA damage, and an increase in the permeability of the mitochondrial membranes (Huang et al., 2010; Lucena et al., 2010). Mitochondrial membrane permeabilization, release of cytochrome $c$, which then binds to Apaf-1, and this complex trigger its oligomerization, forming an apoptosome and recruiting pro-caspase-9. The activated pro-caspase-9 subsequently activates caspase- 3 that cleaved target proteins, leading to the cell apoptosis (Degli Esposti et al., 2012; Wang, 2014).

Superoxide dismutase is involved in the cellular defense against the harmful oxygen species (Pandey et al., 2003), while an increase in MDA production induces the oxidative stress (Monteiro et al., 2006; Kavitha and Rao, 2008; TellezBanuelos et al., 2009). Therefore, a decrease in the SOD activity and increase in the MDA levels represent the markers of hepatocyte oxidative stress, indicating that the critical oxidant -antioxidant balance is disrupted, which can result in hepatocellular ultrastructure alterations and induced apoptosis (Xu et al., 2009). Furthermore, a previous study demonstrated that the oxidative stress may lead to cellular apoptosis (Sinha et al., 2013). The relationship between SOD or MDA and the HEV infection has been rarely investigated. However, in a previous study, significant increase in MDA levels in hepatitis $\mathrm{C}$ virus (HCV) infected patients was observed, and it was demonstrated to be significantly correlated with the HCV RNA viral load. Additionally, MDA levels were proposed to be used as the markers for the monitoring of oxidative stress during $\mathrm{HCV}$ infections (Tawadrous et al., 2012).

Previously, it was demonstrated that the liver-to-body ration increases following the inoculation of animals with $\mathrm{HEV}$ ( $\mathrm{Li}$ et al., 2009; Yang et al., 2015). A significant increase in AST, ALT, and TBIL levels in the sera of Mongolian gerbils was observed, while HEV IgG was detected at day 21 post-inoculation (dpi) (Li et al., 2009; Yang et al., 2015). Ultrastructure analyses demonstrated that the mitochondrial morphology was altered during $\mathrm{HEV}$ infection. However, the molecular mechanisms underlying this injury have not been elucidated. In order to understand the molecular mechanisms of HEV-associated hepatocyte mitochondrial injury pathogenesis, we analyzed the ultrastructure and functional alterations of mitochondria, using Mongolian gerbils infected with HEV.

\section{MATERIALS AND METHODS}

\section{Ethics Statement}

This study was carried out in accordance with the recommendations of ethical guidelines and regulations for the use of laboratory animals by the Animal Care and Use Committee of China Agricultural University (CAU) (approval number: 20140115-089). The protocol was approved by the CAU Animal Care and Use Committee.

\section{HEV Strain}

The strain of HEV, a genotype 4 virus, was derived from the liver sample from a SPF swine infected with HEV (CHN-HBHD-L2, GenBank accession number KM024042). A 10\% (g/mL) homogenate of HEV-positive liver with a titer of $6.57 \times 10^{8}$ genome equivalents (GE) per $\mathrm{mL}$ was prepared (Huang et al., 2009) and tittered using real-time PCR, as previously described (Jothikumar et al., 2006; Zhao et al., 2007; Zwettler et al., 2012; Yang et al., 2015). The obtained homogenate was stored at $-86^{\circ} \mathrm{C}$. All experiments involving viruses were performed in the Animal Bio-safety Level-2 (ABSL-2) facilities. All materials involving viruses were sterilized by autoclaving after use to ensure the inactivation of the viruses.

\section{Animals}

Eighty-four SPF male Mongolian gerbils (Meriones unguiculatus, body weight: 50-60 g; age: 8-10 weeks), were purchased from the Department of Experimental Animal Sciences of Capital Medical University (Beijing, China). To avoid extensive stress to the animals, all experiments were performed following an acclimation period of 3 days after their-arrival to our facility. All animals used in this study were confirmed to be negative for HEV antibodies by ELISA and there were no HEV antigens detected in their sera or fecal samples, according to the nestedPCR analyses, as previously described (Yang et al., 2015). All animals were housed in the SPF facilities.

\section{Experimental Design}

The gerbils were randomized into two groups. Each gerbil in the infected group was intraperitoneally injected with $0.1 \mathrm{~mL}$ of the viral homogenate. Gerbils in the control group were inoculated with the equal volume of homogenate from an SPF swine liver that tested negative for HEV, as previously described (Yang et al., 2015). All gerbils were given food and water ad libitum during the experimental period.

\section{Sampling}

Six gerbils were euthanized to perform necropsy at $0,7,14,21$, 28, 42, and $56 \mathrm{dpi}$. Fresh frozen liver tissues were collected to 


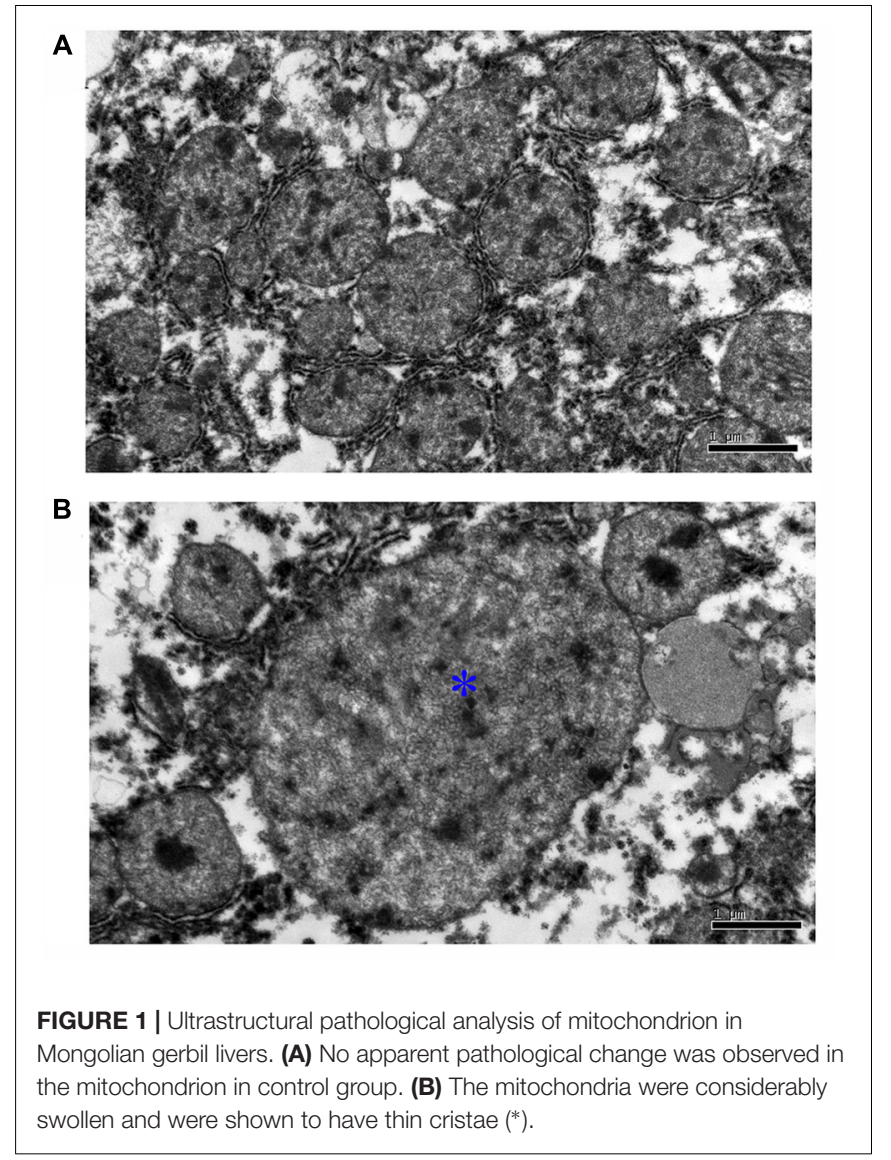

analyze SOD, MDA, and ATPase levels, western-blot and stored at $-86^{\circ} \mathrm{C}$. Additional liver tissue was collected and fixed in neutral $4 \%$ paraformaldehyde for 7 days. The preparation of sections for the TUNEL and immunohistochemical (IHC) analyses were performed, as previous described (Yang et al., 2015). Some of the other tissues were fixed in $2.5 \%(\mathrm{v} / \mathrm{v})$ glutaraldehydepolyoxymethylene solution for 6-8 $\mathrm{h}$ and analyzed using the TEM (Yang et al., 2015).

\section{TEM Analyses}

For the TEM analyses, liver samples were performed as previously described (Yang et al., 2015). These ultra-thin sections were observed using a JEM 100CX TEM.

\section{SOD, MDA, and ATPase Levels in Hepatocytes}

The obtained liver homogenates were centrifuged at $3000 \mathrm{rpm}$ for $10 \mathrm{~min}$ and the supernatants were used to detect SOD and MDA levels. The activity of SOD in hepatocyte was detected by using total SOD (T-SOD) assay kit (hydroxylamine method) (Nanjing Jiancheng Bioengineering Institute). MDA concentrations were detected by using the MDA assay kit (TBA method) (Nanjing Jiancheng Bioengineering Institute), while the ATPase activity was detected by ultramicro-determination ATPase assay kit (Beijing Sino-UK Institute of Biological Technology).

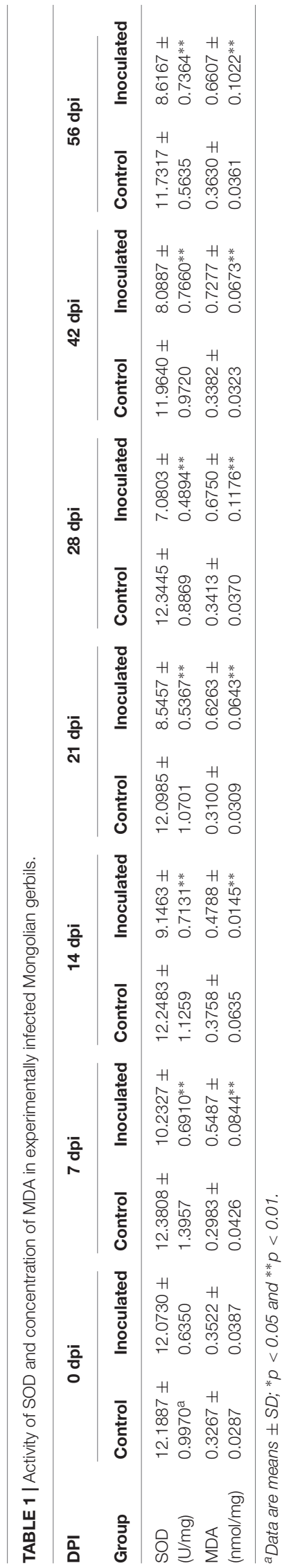



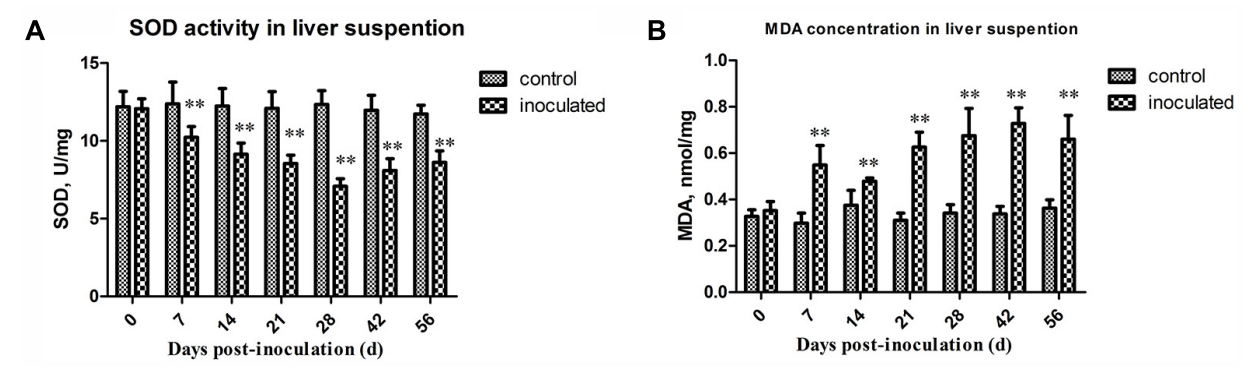

FIGURE 2 | Changes in activity of SOD and concentration of MDA. (A) The activity of SOD decreased significantly in inoculated group compared to that of the control group ( $p<0.01$ ) at 7, 14, 21, 28, 42, and 56 dpi. And at 28 dpi, the activity of SOD was at the lowest level, $7.08 \pm 0.89 \mathrm{U} / \mathrm{mg}$. (B) MDA concentration significantly increased in livers of inoculated group after HEV infection than the control group $(p<0.01)$. And the concentration of MDA was at the peak at 42 dpi, $0.73 \pm 0.07 \mathrm{nmol} / \mathrm{mg} .{ }^{*} p<0.05,{ }^{* *} p<0.01$.

\section{TUNEL Staining}

The apoptotic cells in the livers were detected using TUNEL staining. An in situ detection kit was used according to the manufacturer's instructions (In Situ Cell Death Detection Kit, POD, 11684817910; Roche Biochemicals). The apoptotic indices were calculated as the ratio of positively stained hepatocytes in 100 hepatocytes observed with in one field. Five fields per one sample were analyzed at $400 \times$ magnification).

\section{IHC Assays}

Immunohistochemical staining was performed using a commercial kit, according to the manufacturer's instructions (ZSGB-BIO, Beijing, China). Primary antibodies used in this study were anti-Apaf-1 (1:200, BA2373), anti-Bax (1:300, BA0315), anti-Bcl-2 (1:200, BA0412) and anti-active caspase-3 (1:300, BA3968), anti-active caspase-9 (1:300, BA0690), all obtained from Boster, Co., Ltd., Beijing, China.

Apaf-1, Bax, Bcl-2, and active caspase-3, active caspase- 9 positive signals were observed as brown or yellow granular masses in cells, and their intensities were measured using Motic Med 6.0 CMIAS Image Analysis System (Motic China Group, Co., Ltd., China). A total of 15 fields per gerbil (three fields per section, five sections per gerbil, $400 \times$ magnification) were randomly selected and analyzed. The positive staining intensity was calculated as the ratio of the stained area to the total field assessed (Du et al., 2015).

\section{Western-Blot Analysis}

Small amount of Mongolian gerbil livers obtained from each group were homogenized in the lysis buffer (7 M urea, $2 \mathrm{M}$ thiourea, 4\% CHAPS detergent, 1\% DTT, 400 mM Tris-base, and $1 \mathrm{mM}$ PMSF) (Yang et al., 2015). After centrifugation at $12000 \mathrm{rpm}$ for $20 \mathrm{~min}$ at $4^{\circ} \mathrm{C}$, the supernatants were collected and used as the tissue lysate. Hepatocyte cytoplasmic and mitochondrial proteins were extracted from the liver using Cytoplasmic and Mitochondria Protein Extraction Kit (Sangon Bio-Tech, China) to determine the levels of cytochrome $\mathrm{c}$ in both the cytoplasm and mitochondria of hepatocytes (Pan et al., 2014). Protein concentrations were determined by Nanodrop 2000 spectrophotometer (Thermo, United States). The proteins were separated using sodium dodecyl sulfate polyacrylamide gel electrophoresis (SDS-PAGE) under reducing conditions, and electro-blotted onto polyvinylidene fluoride (PVDF) membranes, as previous described (Yang et al., 2015). The primary antibodies were used including cytochrome c, Apaf-1, Bax, Bcl2 , active caspase-3, active caspase-9, ATP5A1, GAPDH, and $\beta$-actin.

\section{Statistical Analysis}

Experimental data were analyzed using a one-way analysis of variance (ANOVA) with the SPSS20.0 statistical program. The equality of variance was tested and post hoc tests were performed as well. $p<0.05$ was considered statistically significant.

\section{RESULTS}

\section{HEV Infection Induces Mitochondrial Lesion Development}

In the control group, no apparent pathological mitochondrial changes were observed (Figure 1A). However, in the inoculated group, the mitochondria were considerably swollen and were shown to have thin cristae (Figure 1B).

\section{HEV Infection Leads to SOD Activity Decrease and MDA Concentration Increase}

Superoxide dismutase activity and MDA concentration in the investigated animals are presented in Table 1. The activity of SOD was shown to decreased significantly in the inoculated group at $7,14,21,28,42$, and $56 \mathrm{dpi}$, compared with that in the control group $(p<0.01)$ (Figure 2A). At $28 \mathrm{dpi}$, SOD activity was shown to be at the lowest level, $7.08 \pm 0.89 \mathrm{U} / \mathrm{mg}$, in the HEV-infected animals. In contrast, MDA concentrations significantly increased in the livers of the HEV-infected gerbils in comparison with those detected in the control group $(p<0.01$; Figure 2B). MDA concentration peaked at $42 \mathrm{dpi}(0.73 \pm 0.07$ $\mathrm{nmol} / \mathrm{mg})$. 

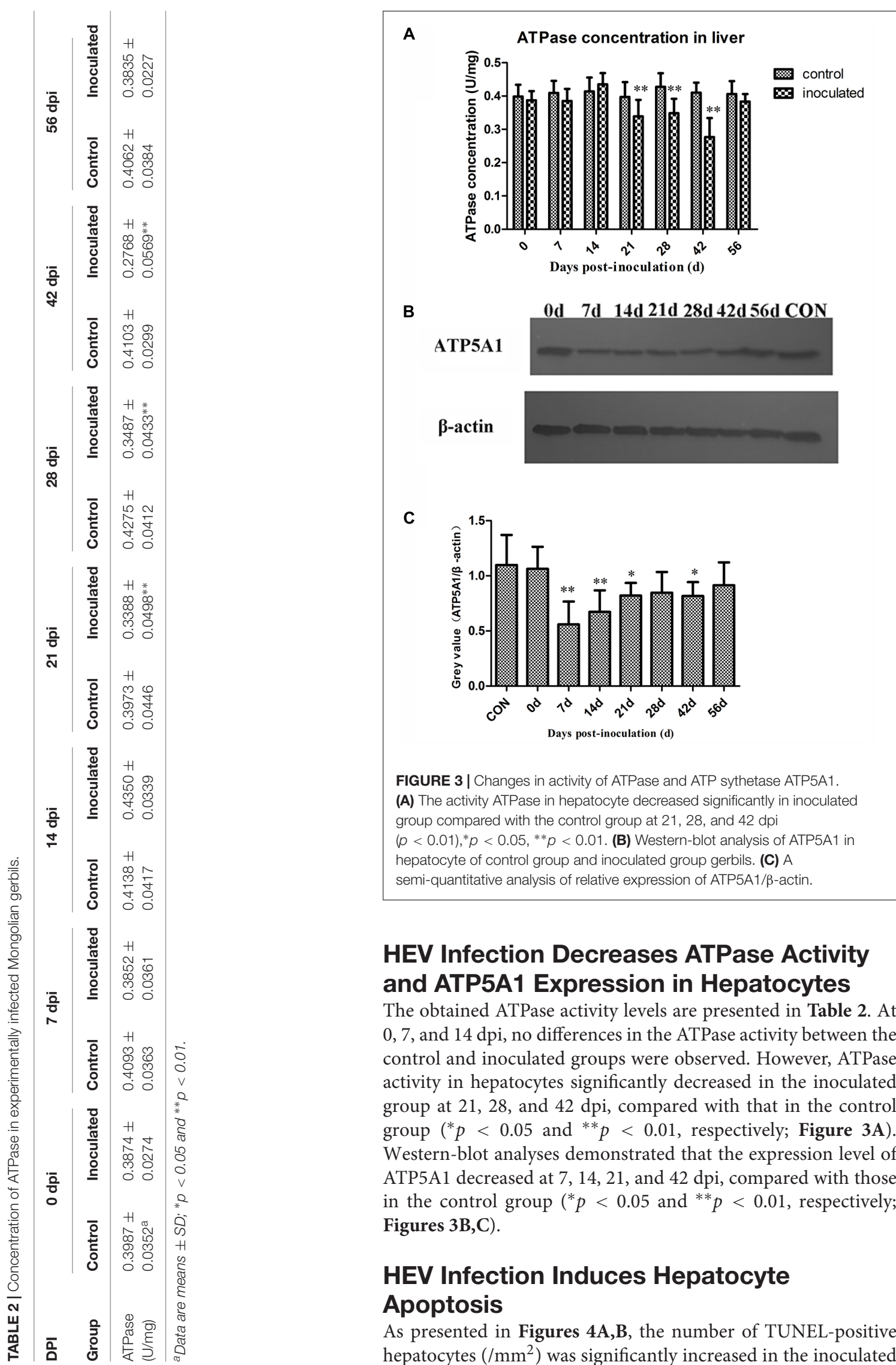

\section{HEV Infection Decreases ATPase Activity and ATP5A1 Expression in Hepatocytes}

The obtained ATPase activity levels are presented in Table 2. At 0,7 , and $14 \mathrm{dpi}$, no differences in the ATPase activity between the control and inoculated groups were observed. However, ATPase activity in hepatocytes significantly decreased in the inoculated group at 21, 28, and $42 \mathrm{dpi}$, compared with that in the control group $\left({ }^{*} p<0.05\right.$ and ${ }^{* *} p<0.01$, respectively; Figure 3A). Western-blot analyses demonstrated that the expression level of ATP5A1 decreased at 7, 14, 21, and $42 \mathrm{dpi}$, compared with those in the control group $\left({ }^{*} p<0.05\right.$ and ${ }^{* *} p<0.01$, respectively; Figures 3B,C).

\section{HEV Infection Induces Hepatocyte Apoptosis}

As presented in Figures $\mathbf{4 A , B}$, the number of TUNEL-positive hepatocytes $\left(/ \mathrm{mm}^{2}\right)$ was significantly increased in the inoculated 

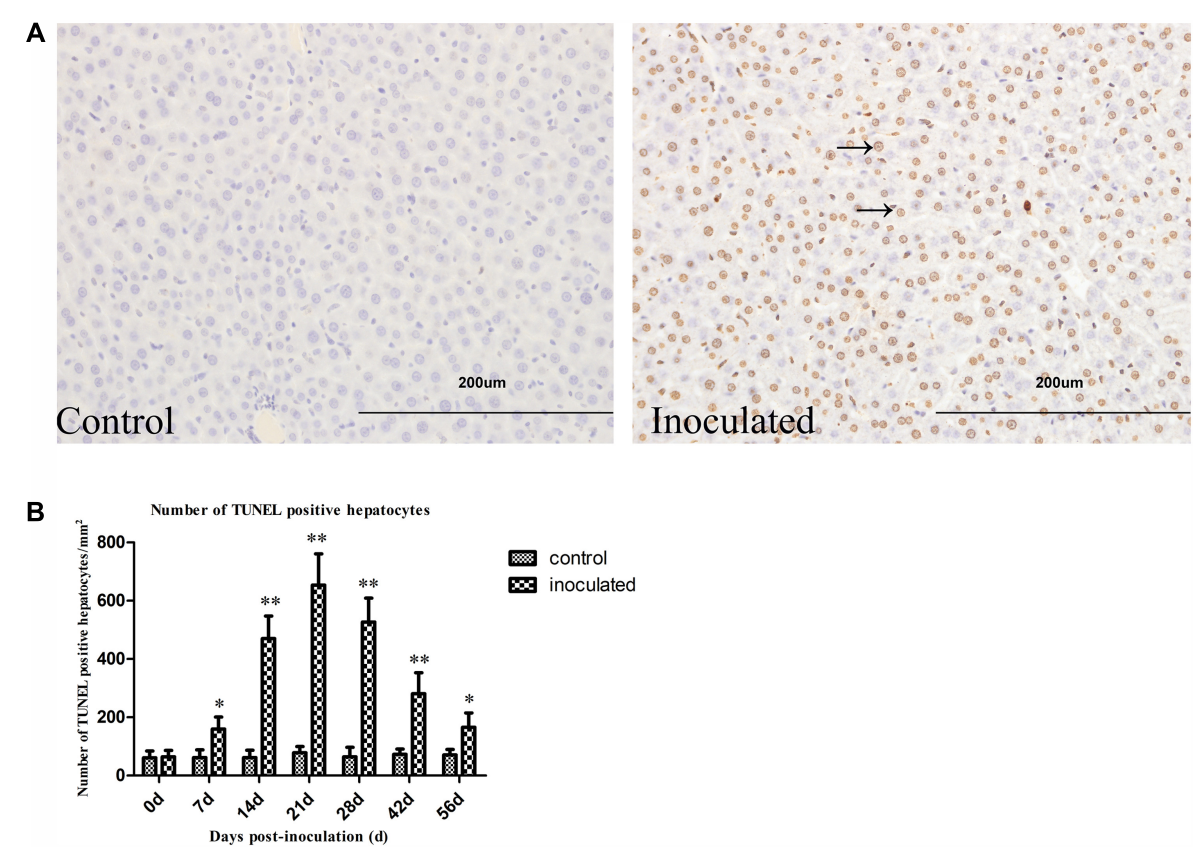

FIGURE 4 | Hepatitis E virus infection induced TUNEL positive hepatocytes increased. (A) Representative pictures of TUNEL staining (arrow indicates TUNEL-positive hepatocytes nuclear stained in brown). (B) Quantitative analysis of number of hepatocyte apoptosis (/mm²). ${ }^{*} p<0.05,{ }^{* *} p<0.01$.

group compared with that in the control group $\left({ }^{*} p<0.05\right.$ and ${ }^{* *} p<0.01$, respectively). The number of TUNEL-positive hepatocytes was shown to increase at $7 \mathrm{dpi}$, and it peaked at 21 dpi.

\section{HEV Infection Induces the Expression of Proteins Regulating Mitochondrion Mediated Apoptosis}

Immunohistochemical staining demonstrated that Bax and Bcl-2 levels considerably increased in the cytoplasm of the hepatocytes in inoculated groups (Figures 5A1,A2,B1,B2). This protein expression up-regulation was confirmed by using the semiquantitative analyses (Figures 5A3,B3). Furthermore, the ratio of Bcl-2 to Bax was calculated from the results obtained in the semiquantitative analyses and they are presented in Table 3. In the inoculated group, Bcl-2 to Bax ratio was below 1 at 14, 21, and 28 $\mathrm{dpi}$, however, in the control group, these ratios were shown to be above 1. Western-blot analyses demonstrated that Bax and Bcl-2 expression levels in the inoculated group were significantly higher than with those in the control group $\left({ }^{*} p<0.05\right.$ and ${ }^{* *} p<0.01$; Figures 5A4,A5,B4,B5).

Therefore, we analyzed cytochrome c levels in the cytoplasm and mitochondria (Figure 5C). Cytochrome c levels in the mitochondria were shown to decrease between 14 and 56 dpi in the inoculated group. In cytoplasm, cytochrome $c$ was initially detected at 7 dpi until the end of the experiment, but it was undetectable at $0 \mathrm{dpi}$ in inoculated group and control group (Figure 5C).

Immunohistochemical staining demonstrated that Apaf-1 expression in the hepatocyte cytoplasm of the inoculated group was significantly higher compared with that in the control group (Figures 6A1,A2). The up-regulation of Apaf1 was confirmed by semi-quantitative analysis $\left({ }^{*} p<0.05\right.$ and ${ }^{* *} p<0.01$; Figure 6A3). Western-blot analyses showed that Apaf-1 expression was significantly higher in the inoculated group, compared with that in the control group $\left({ }^{*} p<0.05\right.$ and ** $p<0.01$; Figures 6A4,A5).

Active caspase-9 and caspase-3 were shown to be expressed at higher levels in the liver of HEV-infected gerbils, compared with those in the control group (Figures 6B1,B2,C1,C2). Similar findings were obtained in the semi-quantitative analyses $\left({ }^{*} p<0.05\right.$ and ${ }^{* *} p<0.01$; Figures 6B3,C3) and westernblot analyses $\left({ }^{*} p<0.05\right.$ and ${ }^{*} p<0.01$; Figures 6B4,B5, C4,C5).

\section{DISCUSSION}

In this study, we determined that HEV infection of Mongolian gerbils may induce the ultrastructural and functional alterations in liver mitochondria, affecting their energy metabolism. ATPase and ATP synthetase, ATP5A1 levels were shown to decrease in the infected animals, together with SOD activity, while the concentration of MDA increased. Taken together, these data demonstrated that HEV infection induces mitochondrial injury, and, consequently, affects, the life cycle of hepatocyte (Everett et al., 2002; Canbay et al., 2004; Amacher, 2005; Guicciardi et al., 2013). TUNEL staining positive demonstrated an increase in apoptotic hepatocyte numbers in the HEV-infected animals, together with the increase in Bax, Bcl-2, Apaf-1, active caspase- 3 and caspase- 9 expression levels. Additionally, cytochrome c levels 
A

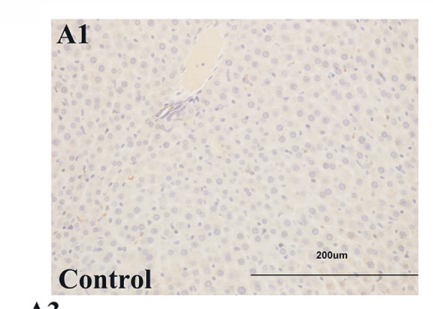

A3

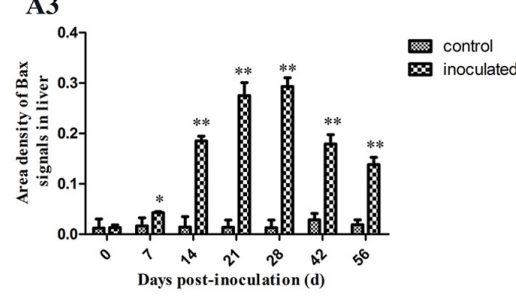

B

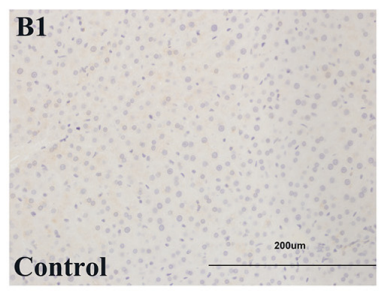

B3

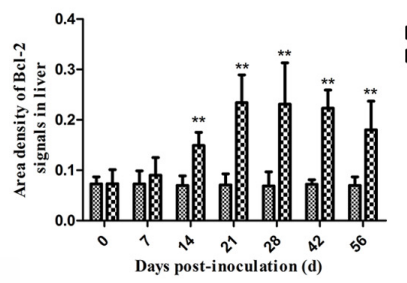

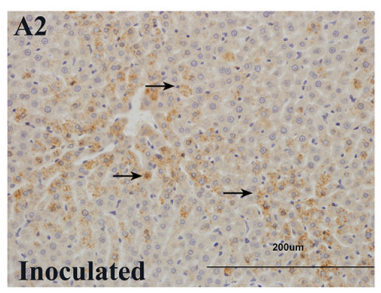

A4

Bax

od 7d 14d 21d 28d 42156dCON

p-actin $-2-2$

A5
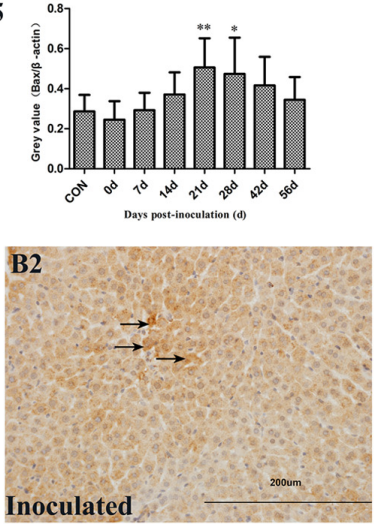

B4

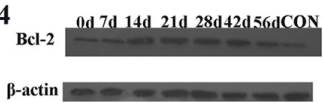

B5

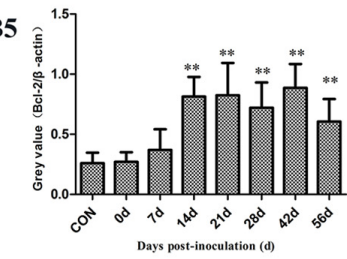

C1

C

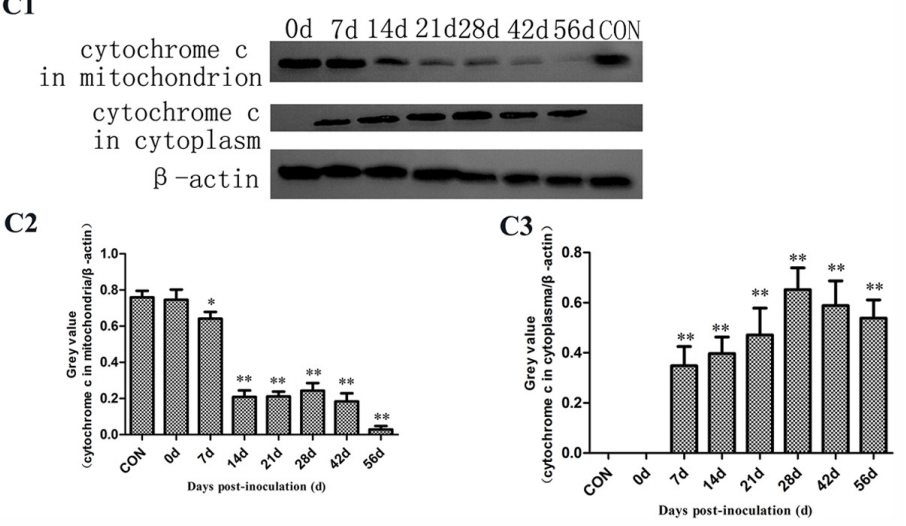

FIGURE 5 | Immunohistochemical and western-blot analysis of Bax, Bcl-2, and cytochrome c proteins. (A1) $\Vdash H C$ analysis of livers in control group, (A2) $\mid H C$ analysis of livers in inoculated group. The primary antibody was Bax antibody, and positive signals were observed in the cytoplasm of hepatocyte $(\rightarrow)$;

(A3) A semi-quantitative analysis of the ratio of Bax positive staining to the total field; (A4) Western-blot analysis of Bax in hepatocyte of control group and inoculated group gerbils; (A5) A semi-quantitative analysis of relative expression of Bax/ $\beta$-actin. (B1) $\mathrm{HC}$ analysis of livers in control group, (B2) $\amalg \mathrm{HC}$ analysis of livers in inoculated group. The primary antibody was Bcl-2 antibody, and the positive signals were observed in the cytoplasm of hepatocyte $(\rightarrow)$; (B3) A semi-quantitative analysis of the ratio of $\mathrm{Bcl}-2$ positive staining to the total field; (B4) Western-blot analysis of $\mathrm{Bcl}-2$ in hepatocyte of control group and inoculated group gerbils; (B5) A semi-quantitative analysis of relative expression of Bcl-2/ $\beta$-actin. (C1) Western-blot analysis of cytochrome $\mathrm{c}$ in mitochondrion and cytoplasm of hepatocyte of control group and inoculated group gerbils. (C2) A semi-quantitative analysis of relative expression of cytochrome $c$ in mitochondria/ $\beta$-actin.

(C3) A semi-quantitative analysis of relative expression of cytochrome c in cytoplasm/ $\beta$-actin. ${ }^{*} p<0.05,{ }^{* *} p<0.01$. 


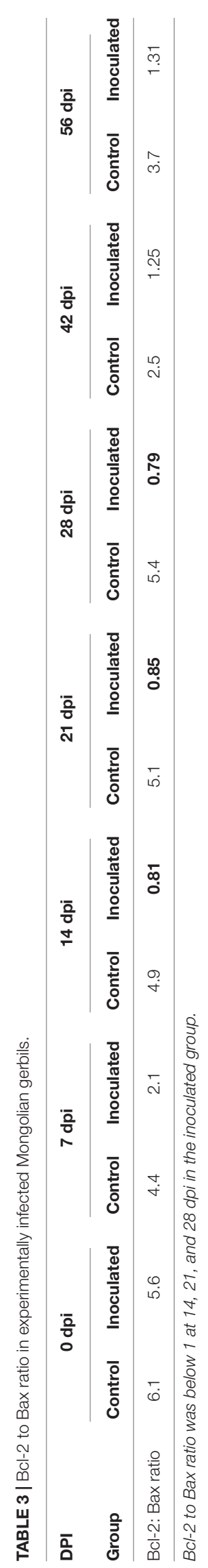

in the cytoplasm of the infected hepatocytes, in contrast to those in the hepatocytes obtained from the control group, showing that HEV infection induces hepatocyte apoptosis via mitochondrial pathway in Mongolian gerbils.

Gerbils were previously successfully infected with HEV, which was then detected in the infected livers, together with and the increased liver enzyme levels (Li et al., 2009; Yang et al., 2015). Here we analyzed the mechanisms underlying these effects and demonstrated that the hepatocyte mitochondria in the HEVinfected gerbils were considerably swollen, with thin cristae, indicating that mitochondria may be targeted during HEV infection.

Since mitochondria play a key role in the apoptotic cascades and cell death (Everett et al., 2002), we further analyzed the expression of mitochondrion-mediated apoptosis-associated proteins.

A previous study demonstrated that hepatocyte apoptosis may be induced by proinflammatory and profibrogenic stimuli, leading to the release of cellular constituents into serum, and liver injury (Canbay et al., 2004). Furthermore, hepatocytes are susceptible to the activation of the mitochondrial apoptotic pathway, in response to excessive free fatty acid generation in the metabolic syndrome (Guicciardi et al., 2013), while the HCV core protein was shown to induce hepatocyte injury and Huh7 cell mitochondrial pathways associated apoptosis (Chou et al., 2005). This pathway is regulated by the Bcl-2 family proteins (Cory and Adams, 2002; Youle and Strasser, 2008), which is why we examined the expression of the members of this family.

The swelling of mitochondrial and thinning of the cristae affects, synthesis and utilization of ATP. ATPase and ATP synthetase ATP5A1 levels in the infected hepatocytes decreased, indicating that HEV infection negatively infects ATP metabolism.

Superoxide dismutase is an important antioxidase, which plays a key role in maintaining the oxidative balance, and its activity levels indirectly reflect the ability of cells to remove free oxygen radicals (Knowles et al., 1969). Furthermore, MDA is a degradation product of lipid peroxidation, which occurs during the interaction between free radicals and poly unsaturated fatty acids (Janero, 1990), and MDA concentrations represent one of the biomarkers of the oxidative stress (Fogelman et al., 1980; Draper and Hadley, 1990). Oxidative stress contributes to the development of many diseases, including some age-related macular degenerations, which are attributed to an inadequately neutralization of oxidative stress (Del Priore et al., 2002; Dunaief et al., 2002; Schutt et al., 2003; Zhou et al., 2005; Weismann et al., 2011), and mitochondrial damage has been associated with oxidative stress related diseases (Chance et al., 1979; Grivennikova et al., 2010). A dysfunction of the mitochondrial antioxidant system may render mitochondria susceptible to injuries despite the adequate cytosolic antioxidant levels (Cano et al., 2014). Viral infections were shown to induce mitochondrial damage and oxidative stress (Acar et al., 2009; Khadem Ansari et al., 2015; Fan et al., 2016), which was confirmed in this study as well, since the SOD activity was shown to decreased while MDA concentrations increased in HEV-infected groups. Our combined results support the conclusion that HEV infection induces mitochondrial damage, which leads to oxidative stress 
A

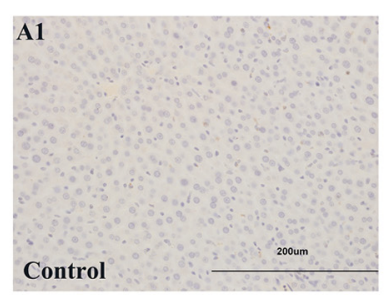

A3

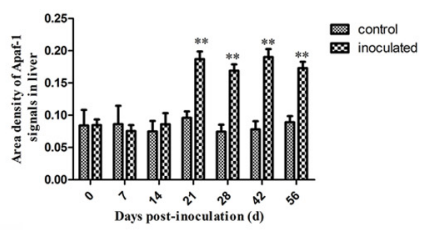

B

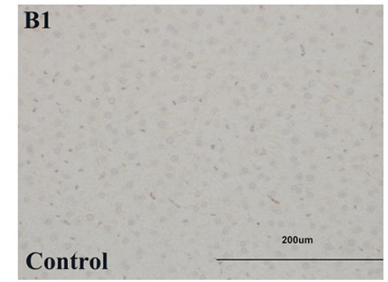

B3

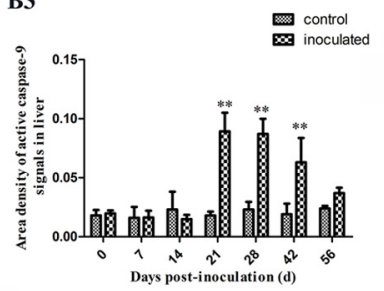

C
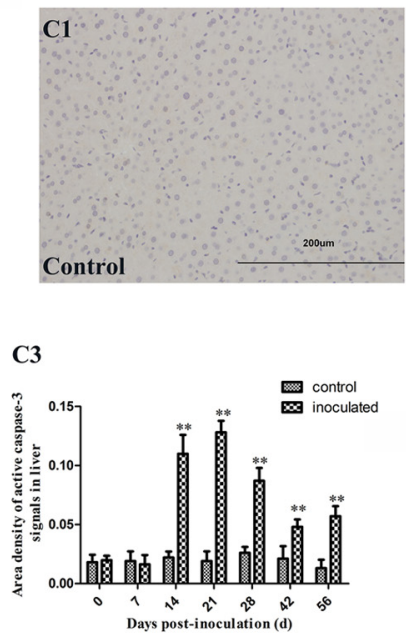

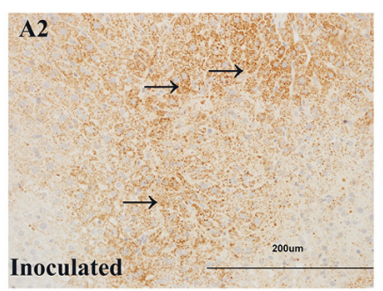

A4

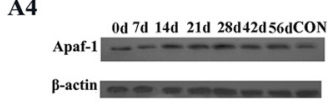

A5
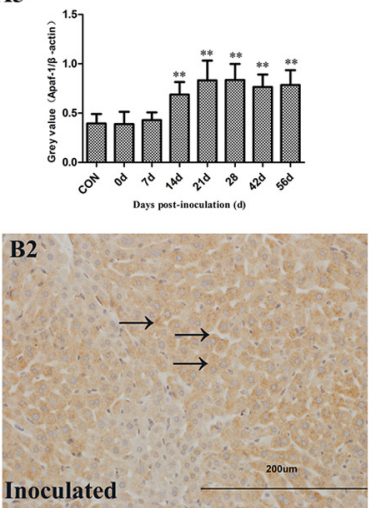

B4

B5
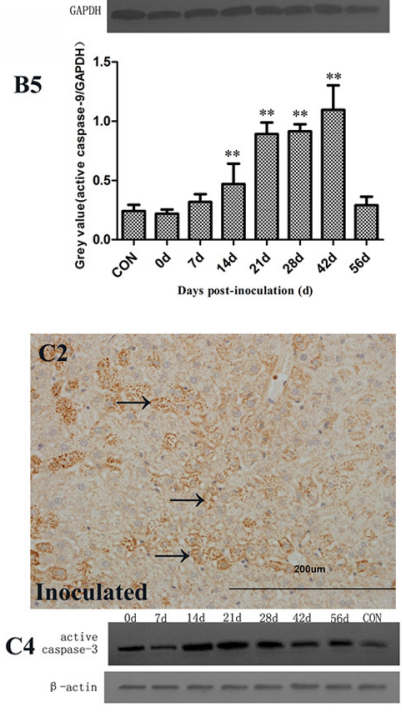

C5

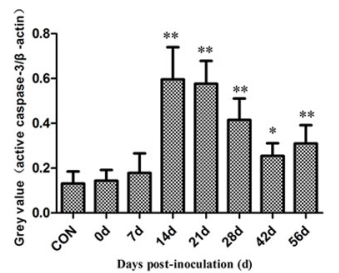

FIGURE 6 | Immunohistochemical and western-blot analysis of Apaf-1, active caspase-9, and active caspase-3 proteins. (A1) IHC analysis of livers in control group, (A2) $\mathrm{IHC}$ analysis of livers in inoculated group. The primary antibody was Apaf-1 antibody, and the positive signals were observed in the cytoplasm of hepatocyte $(\rightarrow)$; (A3) A semi-quantitative analysis of the ratio of Apaf-1 positive staining to the total field; (A4) Western-blot analysis of Apaf-1 protein in hepatocyte of control group 
FIGURE 6 | Continued

and inoculated group gerbils; (A5) A semi-quantitative analysis of relative expression of Apaf- $1 / \beta$-actin. (B1) $\Perp H C$ analysis of livers in control group, (B2) $॥ \mathrm{HC}$ analysis of livers in inoculated group. The primary antibody was active caspase- 9 antibody, and the positive signals were observed in the cytoplasm of hepatocyte ( $\rightarrow$ ); (B3) A semi-quantitative analysis of the ratio of active caspase-9 positive staining to the total field; (B4) Western-blot analysis of active caspase-9 protein in hepatocyte of control group and inoculated group gerbils; (B5) A semi-quantitative analysis of relative expression of active caspase-9/GAPDH. (C1) IHC analysis of livers in control group, (C2) $\Vdash \mathrm{HC}$ analysis of livers in inoculated group. The primary antibody was active caspase-3 antibody, and the positive signals were observed in the cytoplasm of hepatocyte $(\rightarrow)$; (C3) A semi-quantitative analysis of the ratio of active caspase-3 positive staining to the total field; (C4) Western-blot analysis of active caspase-3 protein in hepatocyte of control group and inoculated group gerbils; (C5) A semi-quantitative analysis of relative expression of active caspase-3/ $\beta$-actin. * $p<0.05$, ${ }^{* *} p<0.01$.

development. Mitochondrial dysfunction may be directly or indirectly induced by HEV, and further studies are required to determine the specific mechanisms underlying this process.

To understand the pathogenesis of mitochondrial damage in hepatocytes induced by HEV infection, TUNEL staining was performed, and we determined the levels of proteins associated with the mitochondrial apoptosis pathway. We observed that the number of apoptotic hepatocytes increased in the HEVinfected animals. Furthermore, we analyzed the levels of Bax and Bcl-2, pro-apoptotic and anti-apoptotic proteins, respectively, since the Bax to $\mathrm{Bcl}-2$ ratio plays an important role in the process of cell apoptosis (Adams, 2003). We demonstrated that the expression of $\mathrm{Bcl}-2$ and Bax increased in the infected in the infected group. Bax acts downstream in the mitochondrial apoptotic pathway and it represents a key molecule in this process. As a component of the mitochondrial electron transfer chain, cytochrome c can initiate caspase activation following its release from mitochondria, binding to Apaf-1 (Du et al., 2015). Afterward, Apaf-1-cytochrome c complex forms apoptosome, which recruits procaspase- 9 and initiates the formation of the caspase- 9 holoenzyme that cleaves and activates downstream caspases, such as caspase-3. Furthermore, the recruitment of Bax leads to permeabilization of mitochondrial outer membrane (Wang, 2001; Du et al., 2015), which is considered one of the key control switches of the apoptotic process (Ferreira et al., 2008). Here, we demonstrated that cytochrome $\mathrm{c}$ was released into the cytosol in the inoculated group, and this represents a biomarker indicating the activation of the mitochondrial apoptotic pathway (Pan et al., 2014).

Currently, both animal models and cell cultures are available for HEV pathogenesis investigations. Our team established an efficient Mongolian gerbil model of HEV genotype 4 infection. HEVs were consistently detected in the liver, kidney, and other tissues of Mongolian gerbils, with the characteristic viral hepatitis lesions prominent in the liver. The concentration of AST, ALT, and T-BIL was shown to be significantly increased, while the ultrastructural hepatic injury and anti-HEV IgG positive seroconversion were observed during the infection ( $\mathrm{Li}$ et al., 2009; Yang et al., 2015). HEV RNA was detected in the liver from 7 to $42 \mathrm{dpi}$, consistent with the last days of HEV infection in the swine (Bouwknegt et al., 2009), suggesting that the HEV RNA replication in the Mongolian gerbil was similar to its replication in the swine model. Previously, the activation of mitochondria and caspase- 3 protease expression were shown to be induced, followed by the apoptosis and subsequent necrosis of renal epithelial cells, during the acute phase of HEV infection in Mongolian gerbils. An increased number of apoptotic cells were observed in the renal tubules of these animals, together with the increased levels of Bax, Bcl-2, and caspase- 3 in the kidneys (Soomro et al., 2016). Furthermore, HEVs were detected in the testicular tissues, and the structural and molecular alterations leading to the disruption of the bloodtestis barrier and germ cell apoptosis were observed (Soomro et al., 2017). Using this model, the HEV-4 was demonstrated to be able to cross the blood brain barrier and replicate in the brain and the spinal cord (Shi et al., 2016). Previously, a Z:ZCLA Mongolian gerbil model infected with HEVs isolated from an acute hepatitis E patient was established (Hong et al., 2015). These results are consistent with the observations of this study, and previous studies demonstrated that the Mongolian gerbil represent a promising model for the studies on HEV infection and pathogenesis (Doceul et al., 2016). In vitro, $\mathrm{HEV}$ cultivation was performed using PLC/PRF/5 and A549 using fecal suspension infected with HEV (Tanaka et al., 2007, 2009; Takahashi et al., 2010; Shen et al., 2014), and structural changes to the cells were investigated by TEM, showing that the lysosome numbers increased, indicating apoptosis, and cell membrane was damaged (Shen et al., 2014), which was also consistent with our research.

\section{CONCLUSION}

Taken together, our results demonstrated that HEV infection induces the development of hepatocyte mitochondrion damage in the Mongolian gerbils. Mitochondria were in the oxidative stress situation and were shown to be considerably swollen and their energy metabolism was disrupted. Our investigations demonstrated that $\mathrm{HEV}$ infection activated the mitochondrial apoptotic pathway, which triggered the hepatocyte apoptosis.

\section{AUTHOR CONTRIBUTIONS}

YY and RPS were responsible for the study design. YY, RHS, MS, $\mathrm{FH}$, and FD performed the laboratory work. YY analyzed the data and wrote the article. All authors read, commented on and approved the final article.

\section{FUNDING}

This work was supported by the National Natural Science Foundation of China (Grant Nos. 31702208 and 31472165). 


\section{REFERENCES}

Acar, A., Gorenek, L., Aydin, A., Eyigun, C. P., Eken, A., Sayal, A., et al. (2009). Investigation of oxidative stress and antioxidant defense in patients with hepatitis B virus infection and the effect of interferon-alpha plus lamivudine combination therapy on oxidative stress. Mikrobiyol. Bul. 43, 411-423.

Adams, J. M. (2003). Ways of dying: multiple pathways to apoptosis. Genes Dev. 17, 2481-2495. doi: 10.1101/gad.1126903

Amacher, D. E. (2005). Drug-associated mitochondrial toxicity and its detection. Curr. Med. Chem. 12, 1829-1839. doi: 10.2174/0929867054546663

Bouwknegt, M., Rutjes, S. A., Reusken, C. B., Stockhofe-Zurwieden, N., Frankena, K., de Jong, M. C., et al. (2009). The course of hepatitis E virus infection in pigs after contact-infection and intravenous inoculation. BMC Vet. Res. 5:7. doi: 10.1186/1746-6148-5-7

Canbay, A., Friedman, S., and Gores, G. J. (2004). Apoptosis: the nexus of liver injury and fibrosis. Hepatology 39, 273-278. doi: 10.1002/hep.20051

Cano, M., Wang, L., Wan, J., Barnett, B. P., Ebrahimi, K., Qian, J., et al. (2014). Oxidative stress induces mitochondrial dysfunction and a protective unfolded protein response in RPE cells. Free Radic. Biol. Med. 69, 1-14. doi: 10.1016/j. freeradbiomed.2014.01.004

Chance, B., Sies, H., and Boveris, A. (1979). Hydroperoxide metabolism in mammalian organs. Physiol. Rev. 59, 527-605. doi: 10.1152/physrev.1979.59. 3.527

Chou, A. H., Tsai, H. F., Wu, Y. Y., Hu, C. Y., Hwang, L. H., Hsu, P. I., et al. (2005). Hepatitis C virus core protein modulates TRAIL-mediated apoptosis by enhancing Bid cleavage and activation of mitochondria apoptosis signaling pathway. J. Immunol. 174, 2160-2166. doi: 10.4049/jimmunol.174.4.2160

Cory, S., and Adams, J. M. (2002). The Bcl2 family: regulators of the cellular life-or-death switch. Nat. Rev. Cancer 2, 647-656. doi: 10.1038/nrc883

Degli Esposti, D., Hamelin, J., Bosselut, N., Saffroy, R., Sebagh, M., Pommier, A., et al. (2012). Mitochondrial roles and cytoprotection in chronic liver injury. Biochem. Res. Int. 2012:387626. doi: 10.1155/2012/387626

Del Priore, L. V., Kuo, Y. H., and Tezel, T. H. (2002). Age-related changes in human RPE cell density and apoptosis proportion in situ. Invest. Ophthalmol. Vis. Sci. 43, 3312-3318.

Doceul, V., Bagdassarian, E., Demange, A., and Pavio, N. (2016). Zoonotic hepatitis E virus: classification, animal reservoirs and transmission routes. Viruses 8:E270. doi: 10.3390/v8100270

Draper, H. H., and Hadley, M. (1990). Malondialdehyde determination as index of lipid peroxidation. Methods Enzymol. 186, 421-431. doi: 10.1016/00766879(90)86135-I

Du, F., Ding, Y., Zou, J., Li, Z., Tian, J., She, R., et al. (2015). Morphology and molecular mechanisms of hepatic injury in rats under simulated weightlessness and the protective effects of resistance training. PLOS One 10:e0127047. doi: 10.1371/journal.pone.0127047

Dunaief, J. L., Dentchev, T., Ying, G. S., and Milam, A. H. (2002). The role of apoptosis in age-related macular degeneration. Arch. Ophthalmol. 120, 1435-1442. doi: 10.1001/archopht.120.11.1435

Everett, H., Barry, M., Sun, X., Lee, S. F., Frantz, C., Berthiaume, L. G., et al. (2002). The myxoma poxvirus protein, M11L, prevents apoptosis by direct interaction with the mitochondrial permeability transition pore. J. Exp. Med. 196, 1127-1139. doi: 10.1084/jem.20011247

Fan, X. P., Ji, X. F., Li, X. Y., Gao, S., Fan, Y. C., and Wang, K. (2016). Methylation of the glutathione-S-transferase $\mathrm{P} 1$ gene promoter is associated with oxidative stress in patients with chronic hepatitis B. Tohoku J. Exp. Med. 238, 57-64. doi: $10.1620 /$ tjem. 238.57

Ferreira, R., Neuparth, M. J., Vitorino, R., Appell, H. J., Amado, F., and Duarte, J. A. (2008). Evidences of apoptosis during the early phases of soleus muscle atrophy in hindlimb suspended mice. Physiol. Res. 57, 601-611.

Fogelman, A. M., Shechter, I., Seager, J., Hokom, M., Child, J. S., and Edwards, P. A. (1980). Malondialdehyde alteration of low density lipoproteins leads to cholesteryl ester accumulation in human monocytemacrophages. Proc. Natl. Acad. Sci. U.S.A. 77, 2214-2218. doi: 10.1073/pnas.77. 4.2214

Grivennikova, V. G., Kareyeva, A. V., and Vinogradov, A. D. (2010). What are the sources of hydrogen peroxide production by heart mitochondria? Biochim. Biophys. Acta 1797, 939-944. doi: 10.1016/j.bbabio.2010.02.013
Guicciardi, M. E., Malhi, H., Mott, J. L., and Gores, G. J. (2013). Apoptosis and necrosis in the liver. Compr. Physiol. 3, 977-1010. doi: 10.1002/cphy.c120020

Hong, Y., He, Z. J., Tao, W., Fu, T., Wang, Y. K., and Chen, Y. (2015). Experimental infection of Z:ZCLA Mongolian gerbils with human hepatitis E virus. World J. Gastroenterol. 21, 862-867. doi: 10.3748/wjg.v21.i3.862

Huang, F., Zhang, W., Gong, G., Yuan, C., Yan, Y., Yang, S., et al. (2009). Experimental infection of $\mathrm{Balb} / \mathrm{c}$ nude mice with Hepatitis E virus. BMC Infect. Dis. 9:93. doi: 10.1186/1471-2334-9-93

Huang, J., Shi, W., Zhang, J., Chou, J. W., Paules, R. S., Gerrish, K., et al. (2010). Genomic indicators in the blood predict drug-induced liver injury. Pharmacogenomics J. 10, 267-277. doi: 10.1038/tpj.2010.33

Janero, D. R. (1990). Malondialdehyde and thiobarbituric acid-reactivity as diagnostic indices of lipid peroxidation and peroxidative tissue injury. Free Radic. Biol. Med. 9, 515-540. doi: 10.1016/0891-5849(90) 90131-2

Jothikumar, N., Cromeans, T. L., Robertson, B. H., Meng, X., and Hill, V. R. (2006). A broadly reactive one-step real-time RT-PCR assay for rapid and sensitive detection of hepatitis E virus. J. Virol. Methods 131, 65-71. doi: 10.1016/j. jviromet.2005.07.004

Kavitha, P., and Rao, J. V. (2008). Toxic effects of chlorpyrifos on antioxidant enzymes and target enzyme acetylcholinesterase interaction in mosquito fish, Gambusia affinis. Environ. Toxicol. Pharmacol. 26, 192-198. doi: 10.1016/j.etap. 2008.03.010

Khadem Ansari, M. H., Omrani, M. D., and Kheradmand, F. (2015). Oxidative stress response in patients infected by diverse hepatitis $\mathrm{C}$ virus genotypes. Hepat. Mon. 15:e22069. doi: 10.5812/hepatmon.22069

Knowles, P. F., Gibson, J. F., Pick, F. M., and Bray, R. C. (1969). Electron-spinresonance evidence for enzymic reduction of oxygen to a free radical, the superoxide ion. Biochem. J. 111, 53-58. doi: 10.1042/bj1110053

Kulawiak, B., Hopker, J., Gebert, M., Guiard, B., Wiedemann, N., and Gebert, N. (2013). The mitochondrial protein import machinery has multiple connections to the respiratory chain. Biochim. Biophys. Acta 1827, 612-626. doi: 10.1016/j. bbabio.2012.12.004

Li, W., Sun, Q., She, R., Wang, D., Duan, X., Yin, J., et al. (2009). Experimental infection of Mongolian gerbils by a genotype 4 strain of swine hepatitis $\mathrm{E}$ virus. J. Med. Virol. 81, 1591-1596. doi: 10.1002/jmv.21573

Lill, R., and Mühlenhoff, U. (2008). Maturation of iron-sulfur proteins in eukaryotes: mechanisms, connected processes, and diseases. Annu. Rev. Biochem. 77, 669-700. doi: 10.1146/annurev.biochem.76.052705.162653

Lucena, M. I., Garcia-Martin, E., Andrade, R. J., Martinez, C., Stephens, C., Ruiz, J. D., et al. (2010). Mitochondrial superoxide dismutase and glutathione peroxidase in idiosyncratic drug-induced liver injury. Hepatology 52, 303-312. doi: 10.1002/hep. 23668

Martinou, J.-C., and Youle, R. J. (2011). Mitochondria in apoptosis: Bcl-2 family members and mitochondrial dynamics. Dev. Cell 21, 92-101. doi: 10.1016/j. devcel.2011.06.017

Monteiro, D. A., de Almeida, J. A., Rantin, F. T., and Kalinin, A. L. (2006). Oxidative stress biomarkers in the freshwater characid fish, Brycon cephalus, exposed to organophosphorus insecticide Folisuper 600 (methyl parathion). Comp. Biochem. Physiol. C Toxicol. Pharmacol. 143, 141-149. doi: 10.1016/j. cbpc.2006.01.004

Pan, B., Yang, L., Wang, J., Wang, Y., Wang, J., Zhou, X., et al. (2014). C-Abl tyrosine kinase mediates neurotoxic prion peptide-induced neuronal apoptosis via regulating mitochondrial homeostasis. Mol. Neurobiol. 49, 1102-1116. doi: 10.1007/s12035-014-8646-4

Panda, S. K., Thakral, D., and Rehman, S. (2007). Hepatitis E virus. Rev. Med. Virol. 17, 151-180. doi: 10.1002/rmv.522

Pandey, S., Parvez, S., Sayeed, I., Haque, R., Bin-Hafeez, B., and Raisuddin, S. (2003). Biomarkers of oxidative stress: a comparative study of river Yamuna fish Wallago attu (Bl. \& Schn.). Sci. Total Environ. 309, 105-115. doi: 10.1016/ s0048-9697(03)00006-8

Schutt, F., Bergmann, M., Holz, F. G., and Kopitz, J. (2003). Proteins modified by malondialdehyde, 4-hydroxynonenal, or advanced glycation end products in lipofuscin of human retinal pigment epithelium. Invest. Ophthalmol. Vis. Sci. 44, 3663-3668. doi: 10.1167/iovs.03-0172

Shen, Q., Pu, Y., Fu, X., Xie, Y., Bian, X., Yang, S., et al. (2014). Changes in the cellular proteins of A549 infected with hepatitis E virus by proteomics analysis. BMC Vet. Res. 10:188. doi: 10.1186/s12917-014-0188-5 
Shi, R., Soomro, M. H., She, R., Yang, Y., Wang, T., Wu, Q., et al. (2016). Evidence of Hepatitis E virus breaking through the blood-brain barrier and replicating in the central nervous system. J. Viral Hepat. 23, 930-939. doi: 10.1111/jvh.12557

Sinha, K., Das, J., Pal, P. B., and Sil, P. C. (2013). Oxidative stress: the mitochondriadependent and mitochondria-independent pathways of apoptosis. Arch. Toxicol. 87, 1157-1180. doi: 10.1007/s00204-013-1034-4

Smith, D. B., Simmonds, P., Jameel, S., Emerson, S. U., Harrison, T. J., Meng, X. J., et al. (2014). Consensus proposals for classification of the family Hepeviridae. J. Gen. Virol. 95(Pt 10), 2223-2232. doi: 10.1099/vir.0.068429-0

Soomro, M. H., Shi, R., She, R., Yang, Y., Hu, F., and Li, H. (2016). Antigen detection and apoptosis in Mongolian gerbil's kidney experimentally intraperitoneally infected by swine hepatitis E virus. Virus Res. 213, 343-352. doi: 10.1016/j.virusres.2015.12.012

Soomro, M. H., Shi, R., She, R., Yang, Y., Wang, T., Wu, Q., et al. (2017). Molecular and structural changes related to hepatitis $\mathrm{E}$ virus antigen and its expression in testis inducing apoptosis in Mongolian gerbil model. J. Viral Hepat. 24, 696-707. doi: $10.1111 /$ jvh. 12690

Takahashi, M., Tanaka, T., Takahashi, H., Hoshino, Y., Nagashima, S., Jirintai, et al. (2010). Hepatitis E virus (HEV) strains in serum samples can replicate efficiently in cultured cells despite the coexistence of HEV antibodies: characterization of HEV virions in blood circulation. J. Clin. Microbiol. 48, 1112-1125. doi: 10.1128/jcm.02002-09

Tanaka, T., Takahashi, M., Kusano, E., and Okamoto, H. (2007). Development and evaluation of an efficient cell-culture system for Hepatitis E virus. J. Gen. Virol. 88(Pt 3), 903-911. doi: 10.1099/vir.0.82535-0

Tanaka, T., Takahashi, M., Takahashi, H., Ichiyama, K., Hoshino, Y., Nagashima, S., et al. (2009). Development and characterization of a genotype 4 hepatitis $\mathrm{E}$ virus cell culture system using a HE-JF5/15F strain recovered from a fulminant hepatitis patient. J. Clin. Microbiol. 47, 1906-1910. doi: 10.1128/jcm.006 29-09

Tawadrous, G. A., Aziz, A. A., Amin, D. G., Eldemery, A., and Mostafa, M. A. (2012). RANTES, TNF-alpha, oxidative stress, and hematological abnormalities in hepatitis C virus infection. J. Investig. Med. 60, 878-882. doi: 10.2310/JIM. 0b013e318254519e

Tellez-Banuelos, M. C., Santerre, A., Casas-Solis, J., Bravo-Cuellar, A., and Zaitseva, G. (2009). Oxidative stress in macrophages from spleen of Nile tilapia (Oreochromis niloticus) exposed to sublethal concentration of endosulfan. Fish Shellfish Immunol. 27, 105-111. doi: 10.1016/j.fsi.2008. 11.002
Wang, K. (2014). Molecular mechanisms of hepatic apoptosis. Cell Death Dis. 5:e996. doi: 10.1038/cddis.2013.499

Wang, X. (2001). The expanding role of mitochondria in apoptosis. Genes Dev. 15, 2922-2933.

Weismann, D., Hartvigsen, K., Lauer, N., Bennett, K. L., Scholl, H. P., Charbel Issa, P., et al. (2011). Complement factor $\mathrm{H}$ binds malondialdehyde epitopes and protects from oxidative stress. Nature 478, 76-81. doi: 10.1038/nature10449

Xu, W. N., Liu, W. B., and Liu, Z. P. (2009). Trichlorfon-induced apoptosis in hepatocyte primary cultures of Carassius auratus gibelio. Chemosphere 77, 895-901. doi: 10.1016/j.chemosphere.2009.08.043

Yang, Y., Shi, R., She, R., Soomro, M. H., Mao, J., Du, F., et al. (2015). Effect of swine hepatitis E virus on the livers of experimentally infected Mongolian gerbils by swine hepatitis E virus. Virus Res. 208, 171-179. doi: 10.1016/j.virusres.2015. 06.007

Youle, R. J., and Strasser, A. (2008). The BCL-2 protein family: opposing activities that mediate cell death. Nat. Rev. Mol. Cell Biol. 9, 47-59. doi: 10.1038/nrm2308

Zhao, C., Li, Z., Yan, B., Harrison, T. J., Guo, X., Zhang, F., et al. (2007). Comparison of real-time fluorescent RT-PCR and conventional RT-PCR for the detection of hepatitis E virus genotypes prevalent in China. J. Med. Virol. 79, 1966-1973. doi: 10.1002/jmv.21040

Zhou, J., Cai, B., Jang, Y. P., Pachydaki, S., Schmidt, A. M., and Sparrow, J. R. (2005). Mechanisms for the induction of HNE- MDA- and AGE-adducts, RAGE and VEGF in retinal pigment epithelial cells. Exp. Eye Res. 80, 567-580. doi: 10.1016/j.exer.2004.11.009

Zwettler, D., Fink, M., Revilla-Fernandez, S., Steinrigl, A., Winter, P., and Kofer, J. (2012). First detection of hepatitis E virus in Austrian pigs by RT-qPCR. Berl. Munch. Tierarztl. Wochenschr. 125, 281-289.

Conflict of Interest Statement: The authors declare that the research was conducted in the absence of any commercial or financial relationships that could be construed as a potential conflict of interest.

Copyright (c) 2018 Yang, Shi, Soomro, Hu, Du and She. This is an open-access article distributed under the terms of the Creative Commons Attribution License (CC BY). The use, distribution or reproduction in other forums is permitted, provided the original author(s) and the copyright owner are credited and that the original publication in this journal is cited, in accordance with accepted academic practice. No use, distribution or reproduction is permitted which does not comply with these terms. 